\title{
A Privacy-Preserving Device Tracking System Using a Low-Power Wide-Area Network
}

\author{
Tomer Ashur ${ }^{1}$, Jeroen Delvaux ${ }^{1}$, Sanghan Lee ${ }^{2}$, Pieter Maene ${ }^{1}$, Eduard Marin ${ }^{1}$, \\ Svetla Nikova ${ }^{1}$, Oscar Reparaz ${ }^{1}$, Vladimir Rožićc ${ }^{1}$, Dave Singelée ${ }^{1}$, Bohan Yang ${ }^{1}$, \\ and Bart Preneel ${ }^{1}$ \\ 1 imec-COSIC KU Leuven, Belgium \\ firstname. lastname@esat.kuleuven.be \\ 2 The Attached Institute of ETRI \\ 1559, Yuseong-daero, Yuseong-gu, Daejeon, 34044, South Korea \\ freewill71@nsr.re.kr
}

\begin{abstract}
This paper presents the design and implementation of a lowpower privacy-preserving device tracking system based on Internet of Things (IoT) technology. The system consists of low-power nodes and a set of dedicated beacons. Each tracking node broadcasts pseudonyms and encrypted versions of observed beacon identifiers over a Low-Power WideArea Network (LPWAN). Unlike most commercial systems, our solution ensures that the device owners are the only ones who can locate their devices. We present a detailed design and validate the result with a prototype implementation that considers power and energy consumption as well as side-channel attacks. Our implementation uses Physically Unclonable Function (PUF) technology for secure key-storage in an innovative way. We build and evaluate a complete demonstrator with off-the-shelf IoT nodes, Bluetooth Low Energy (BLE) beacons, and LoRa long distance communication (LPWAN). We validate the setup for a bicycle tracking application and also estimate the requirements for a low-cost ASIC node.
\end{abstract}

\section{Introduction}

The Internet of Things (IoT) will transform our society. Predictions about the scale and speed of this transformation vary - Gartner claims that there will be 26 billion IoT devices by 2020, Cisco predicts 50 billion, Intel 200 billion and IDC 212 billion - but there is no doubt that this development will have a major impact on our lives.

One of the main benefits of the IoT is that it links the physical world to the online world. A key application is the localization and tracking of objects. The simplest way to track objects is by adding a barcode. However, those are limited to the line of sight of the reader and store little information. RadioFrequency IDentification (RFID) tags overcome these limitations; they were mostly considered for deployment in warehouses or shops, but with the appropriate back-office infrastructure, they could be used for tracking during the complete supply chain. Unfortunately, RFID tags require external power, have only limited computational capabilities, and can only be used for short-range communications. Another development is the tracking of more sophisticated mobile devices such as smartphones, tablets, and laptops. The first two typically have Global Positioning System (GPS) receivers and all of them can detect nearby WiFi networks. Using 
an Internet connection, it is rather straightforward to make them 'phone home' so that legitimate owners can recover them. A similar feature is being added to cars, in order to assist users to find their car in large parking lots, or to help track lost or stolen cars. Multiple location services allow users to locate their friends or spouses using smartphones. Another fascinating development created by modern technologies is participatory sensing: while the original goal was to crowdsource data on weather or mobility patterns, they could also be used to track objects or users. Dedicated IoT trackers are also arriving on the market: low-cost nodes broadcast information that helps owners locate their keys, wallets, or bicycles. Some of these devices such as Tile [6] and TrackR [7] use a combination of Bluetooth Low Energy (BLE) with crowdsourcing (GPS location on smartphones); others such as SemTech exploit the fact that - unlike RFID tags - modern IoT nodes can communicate over long distances with a Low-Power Wide-Area Network (LPWAN) such as LoRa. For several devices, vulnerabilities have been reported, e.g., in [4].

With the deployment of these applications, there has been an increased interest in the privacy aspects of tracking, and in a broader sense, in the privacy of location-based services. At first sight, it seems to be impossible to reconcile these properties: devices should be tracked by their legitimate owners, yet one wants to offer privacy against third parties and service providers. Cryptographic techniques can overcome this paradox. In particular in the area of RFID, several solutions have been proposed that offer a broad range of tradeoffs between security, privacy, and scalability. However, low-cost IoT devices have capabilities that go well beyond those of RFID tags. While one can repurpose some of the ideas, long distance communications bring new threats but also new opportunities to enhance security and privacy. For devices that are connected to the Internet, more sophisticated solutions have been developed that offer advanced privacy features (e.g., the Adeona and Eddystone systems described in Sect. 2). We show how similar goals can be achieved with low-energy and low-cost nodes and for devices that are not inherently connectable.

Contributions. We present a system that allows users to track the location of their devices such that the intermediate entities learn neither the device's location nor to whom it belongs. Our privacy-preserving tracking system is based on a small, low-cost tracking device, also known as a tag, that is attached to any user's personal belongings (e.g., a bicycle), and a set of dedicated beacons. A schematic overview of the system is depicted in Fig. 1. Our protocol provides message confidentiality and integrity as well as entity authentication, unlinkability, and forward privacy. Furthermore, it has built-in resistance to side-channel attacks. Our prototype uses commercial components and offers secure key storage based on a PUF. The latency of our prototype, as well as its power and energy consumption, is evaluated, and we also outline an ASIC design for the tag.

Notation. Binary vectors are denoted by a bold-faced, lowercase character, e.g., $\mathbf{x}=\left(x_{1} x_{2}\right)$. All vectors are row vectors. The all-zeros vector is denoted by $\mathbf{0}$ Binary matrices are denoted by a bold-faced, uppercase character, e.g., X. A random variable is denoted by a regular uppercase character, e.g., $X$. The set of all $n$-bit vectors is denoted by $\{0,1\}^{n}$. Procedure names are printed in a sans-serif font, e.g., Func(·). The operator $\|$ denotes concatenation. 


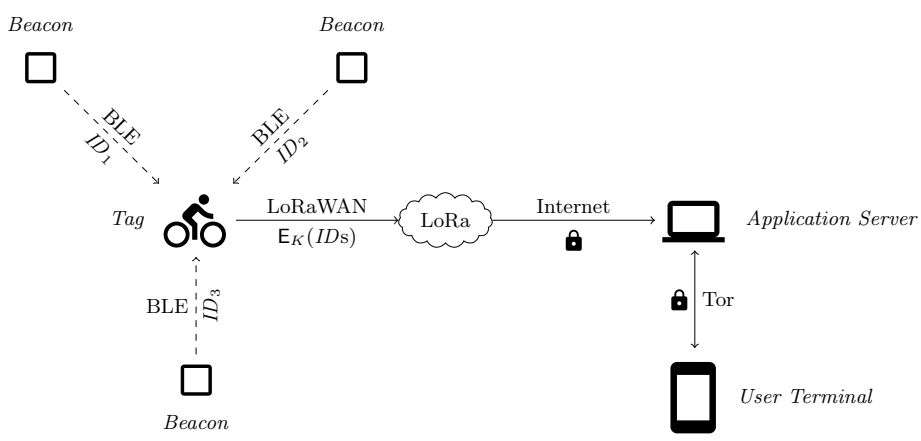

Fig. 1: The proposed tracking system showing all components and communication channels involved.

Organization. The remainder of this paper is organized as follows. Section 2 gives an overview of the related work. Section 3 details the threat model and assumptions about the building blocks. Section 4 describes the design requirements and the proposed protocol, whereas the practical aspects and the implementation of our system are discussed in Sect. 5. The security and privacy are analyzed in Sect. 6. An evaluation of our prototype implementation is given in Sect. 7 . Finally, Sect. 8 presents concluding remarks.

\section{Related Work}

Over the last years, the problem of providing privacy in tracking systems has been extensively studied in RFID systems. See for instance the series of papers by Avoine et al. [15,14,20,18,19,17], Juels et al. [38,37], Weis et al. [54], Molnar et al. [47], Henrici et al. [34,33,35], Saito et al. [50], Alomair et al. [12,22] and Spiekermann et al. [52].

Variants of this problem arise in other technologies such as electronic passports [16] or Bluetooth-based localization services. Google's Eddystone [32] is a modern example of a BLE-based tracking system featuring some privacy enhancements. Eddystone allows "ephemeral identifiers" that rotate periodically to enhance privacy. Only trusted parties can resolve the ephemeral identifier to the actual identity by performing an expensive, yet feasible, computation. Other parties that attempt to deanonymize ephemeral identifiers are faced with a computational problem of prohibitive cost. Apple's iBeacon is a BLE-based tracking system but it does not support any equivalent of ephemeral identifiers [9].

In addition to BLE, key technologies for location tracking are GPS and RFID. The former is a satellite-based positioning system providing $3 \mathrm{D}$ coordinates with up to five-meter-precision anywhere on Earth. This high accuracy, however, comes at a cost in terms of power, with modern GPS receivers drawing up to tens of $\mathrm{mA}$ when tracking objects [8]. RFID tags are passively powered circuits that are typically used to identify objects. Due to their passive nature, the power consumption of these tags is extremely low, with read-outs requiring only around $10 \mu \mathrm{W}$ of input power from the reader [11]. However, this also implies that tags can only be read out from a limited distance, therefore offering a shorter 
range and a lower accuracy than GPS. In our system, the tag determines its position using BLE, which has a current draw on the order of hundreds of $\mu \mathrm{A}$ when scanning for beacons [10]. Our solution can therefore be positioned between GPS and RFID in terms of range, accuracy, and power.

Several systems have been proposed that allow tracking of objects such as bicycles, while using existing IoT technologies and preserving the user's privacy. Bochem et al. [25] presented a privacy-preserving tracking system that relies on GPS-enabled devices and base stations distributed over the tracking area. When a device is reported as stolen by its legitimate user, base stations broadcast its ID in order to activate its tracking module. This solution, however, presents several limitations. First, broadcasting the unique ID of a stolen device can pose significant privacy risks. Second, GPS may not be suitable for resource-constrained tracking modules since it incurs high energy costs. Third, this solution requires the service provider to be trusted. A rogue service provider could, for example, pretend that a given device has been reported as stolen and start broadcasting its ID. Fourth, the device needs to support downlink communication with the base stations. This process can be expensive in terms of energy since the device needs to periodically scan for messages sent by base stations. We address all four limitations: tags encrypt beacon IDs and use pseudonyms, localization is achieved using BLE, the service provider is trusted only for availability reasons, and tags only use uplink communication.

The Adeona system by Ristenpart et al. [49] presents an efficient solution that can be used to track Internet-connected devices. However, Adeona has several limitations. Firstly, it only supports the tracking of devices that are connected to the Internet. Secondly, the location is determined using IP geolocation services. Thirdly, it requires devices and users to have a synchronized clock. Our solution addresses all these shortcomings. By using BLE and LoRa technologies, objects that are isolated from the Internet can be tracked without the need for a synchronized clock.

\section{Threat Model and Assumptions}

Threat Model. Our privacy-preserving tracking system considers the presence of both passive adversaries, who can eavesdrop on any given communication channel in Fig. 1, and active adversaries, who can additionally jam, replay, modify, inject, delay, and forge messages. We do not consider fingerprinting attacks that uniquely identify a device based on its physical characteristics [28]. Adversaries can obtain physical access to a tag and perform simple side-channel attacks such as Simple Power Analysis (SPA) and Differential Power Analysis (DPA). More sophisticated side-channel attacks such as template attacks require extensive profiling of the device and are not considered. We do not claim any resistance to adversaries who can perform physically invasive attacks on a tag. This implies that data stored in volatile memory, e.g., registers, and Non-Volatile Memory (NVM), e.g., flash, can be neither read nor modified. In Sect. 4.4, we extend our design such that the assumption of secure NVM can be relaxed. Debugging and programming interfaces are irreversibly disabled, i.e., internal components cannot be accessed through software tools. Adversaries can detach tags from their assets; for a bicycle, this could be made more difficult by hiding tags inside the frame. 
Finally, note that an adversary could always track an object in a limited region with relatively modest means, e.g., a set of cameras.

Assumptions. While some of the beacons can act maliciously, e.g., by broadcasting the ID of another beacon, we assume that the majority of the beacons is legitimate. This enables the user terminal to detect inconsistencies in the received location data. The LoRa infrastructure is trusted only for availability reasons; LoRa provides built-in device authentication which allows to protect against Denial-of-Service (DoS) attacks such as flooding. However, the current LoRa protocol does not provide any location privacy since all messages contain the unique Media Access Control (MAC) address of the sender. Our solution targets a future, privacy-preserving version of LoRaWAN that would support privacy features such as MAC randomization. Furthermore, while we choose LoRa to build our prototype, any similar networking technology could be used.

The application server is trusted for availability reasons and is assumed to be honest-but-curious. In other words, it follows the protocol specifications but may try to learn information about the users and the tags. The application server communicates with both the LoRa infrastructure and the user terminal over a secure channel, e.g., using Transport Layer Security (TLS). To preserve anonymity, users can establish a connection over the Internet with, e.g., Tor [30]. Moreover, users should regularly delete their tag's location history from their terminal, thereby limiting forward privacy loss if the terminal were to be compromised.

\section{Design}

Sect. 4.1 describes the main entities and communication channels of our proposed system. The requirements of the corresponding protocol are listed in Sect. 4.2, and followed by its specification in Sect. 4.3. To allow for more cost-effective NVM technologies with relaxed assumptions on physical security, we extend our design with a PUF-based key generator in Sect. 4.4.

\subsection{System Model}

Our privacy-preserving tracking system is shown in Fig. 1 and can be summarized as follows: a tag scans the BLE channel at fixed time intervals and stores the IDs of all beacons it sees. In addition, the tag periodically sends an encrypted list of observed beacon IDs to the application server via LoRa. LoRa is currently one of the most promising wireless technologies, because it allows for long-range, low-power, and low-cost communication. This makes LoRa suitable for tracking devices that need to transmit small amounts of data a few times per day over long distances. It is important to note, however, that other LPWAN technologies could be used as well.

To prevent unauthorized parties from linking LoRa messages to tags, the use of a public and static tag ID should be avoided. In our proposal, tags reveal their identity through a one-time pseudonym that is refreshed with every message and that can only be regenerated by the user terminal. By querying the application server with a valid pseudonym, the user terminal can retrieve the location of its tracking device. 
Below, we describe the five main entities of our system in more detail. This includes tags, BLE beacons, the LoRa infrastructure, the application server, and the user terminal.

Tags. Tags are small, inexpensive, low-energy, and self-powered devices that are attached to an object, e.g., a bicycle, for tracking purposes. They consist of a low-end microcontroller and the following two network interfaces: BLE and LoRaWAN. The former is used to collect the unique ID of all beacons that are in close proximity to the tag, whereas the latter is used to regularly send the encrypted version of the beacon IDs to the application server.

Beacons. Battery-powered beacons are provisioned in tracking-enabled areas. Each beacon continuously transmits its unique ID over BLE.

LoRa Infrastructure. The LoRa infrastructure includes gateways and servers that are managed by a network provider. The main function of the LoRa infrastructure is to act as a channel between the tags and the application server. More specifically, the LoRa infrastructure uses gateways to gather the messages sent by tags over LoRa, stores them on internal servers, and then forwards them to the corresponding application server.

Application Server. The application server collects the encrypted versions of beacon IDs, which are sent by the tags through the LoRa infrastructure, and provides them upon request to the authorized users.

User Terminal. Users download the location history of their tags from the application server. The user terminal can be a smartphone or a laptop.

Beacons communicate with tags wirelessly using a unidirectional BLE channel, whereas the communication between tags and the LoRa infrastructure takes place over a LoRa uplink. The application server communicates with the LoRa infrastructure and the user terminal over the Internet, respectively using a secure channel (e.g. TLS) and through an anonymous network (e.g. Tor).

\subsection{Design Requirements}

Our privacy-preserving tracking system should satisfy the following functional $(\mathrm{F})$, security $(\mathrm{S})$ and privacy $(\mathrm{P})$ requirements.

(F1) Energy Usage: A tag's energy usage for both transmissions and computations should be as low as possible.

(F2) Cost: The cost of tags after adding the security and privacy mechanisms should remain as low as possible.

(F3) Efficiency: Users should be able to retrieve the location data from the application server in an efficient manner.

(S1) Confidentiality of Location Data: Only authorized users should be able to access the location history of their tags.

(S2) Message Integrity: Users should be assured that the received messages are fresh and have not been altered during transit.

(S3) Tag Authentication: Users should be assured that the received messages are sent by the tags attached to their personal belongings.

(P1) Tag Identity Privacy: No unauthorized entity should be able to learn the identity of a tag from its transmissions.

(P2) Message Unlinkability: No entity should be able to link a transmission to a tag, or even associate two transmissions to the same source.

(P3) Forward Privacy: Adversaries who compromise a tag or its corresponding user terminal should not be able to learn the tag's past locations. 


\subsection{Protocol}

We now specify a protocol that allows users to track their belongings in a privacypreserving manner. At constant time intervals, a tag wakes up from sleep mode and stores all beacon IDs it can receive over BLE. The geographical coordinates of each beacon are known and hence allow users to estimate the location of their tags. For this purpose, each tag periodically transmits an encrypted version of the collected beacon IDs to the application server over LoRa, using a key that is shared with the legitimate user only. Each message also contains an ID of the tag and can hence be retrieved by the user. Given that adversaries, including the application server, would be able to link messages if tag IDs are static, this comprises a dynamic, one-time pseudonym that can be reconstructed by the user terminal only. To prevent linkage through predicable transmission times, each message is sent at a random time within a pre-determined interval.

Following the manufacturing of a tag, a state stored in physically secure NVM is initialized with a tag-specific, symmetric master key $\mathbf{k}_{\mathrm{Tag}}$. The programming interface is irreversibly disabled afterwards. The value of $\mathbf{k}_{\text {Tag }}$ is also printed in the form of a machine-readable barcode, i.e., a Quick Response (QR) code [36], and given to the user when the tag is bought. To prevent the key $\mathbf{k}_{\mathrm{Tag}}$ from leaking through the supply chain, the QR code is stored inside the tracker's tamper-evident packaging. The user terminal is initialized by scanning the $\mathrm{QR}$ code and hence shares $\mathbf{k}_{\mathrm{Tag}}$ with the tag. After the initialization, the QR code should be either destroyed or stored in, e.g., the user's home safe. To facilitate an implementation resistant to side-channel attacks (cf. Sect. 6.2), a tag updates the value of its key after every transmission. The first session key $\mathbf{k}_{0}$ is obtained by feeding $\mathbf{k}_{\text {Tag }}$ into a cryptographic hash function $\mathrm{H}$, thereby overwriting the state stored in NVM. As depicted in Fig. 2, the same update mechanism applies to all subsequent session keys $\mathbf{k}_{i}$.

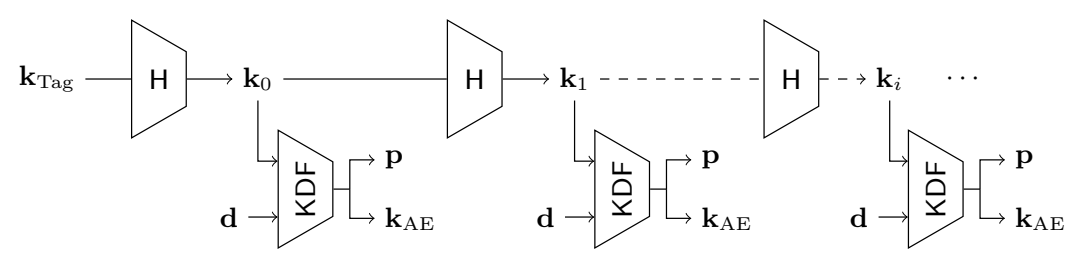

Fig. 2: A one-time session key $\mathbf{k}_{i}$ is obtained by hashing the previous session key $\mathbf{k}_{i-1}$, with $\mathbf{k}_{0}=\mathrm{H}\left(\mathbf{k}_{\mathrm{Tag}}\right)$. Next, the pseudonym $\mathbf{p}$ and encryption key $\mathbf{k}_{\mathrm{AE}}$ are derived from $\mathbf{k}_{i}$. Here $\mathbf{H}$ is a cryptographic hash function and $\operatorname{KDF}(\mathbf{d}, \mathbf{k})$ a Key Derivation Function with key domain separator $\mathbf{d}$.

To protect a user's privacy, we designed our system such that tags only transmit a single LoRa message per hour and the LoRa network provider does not learn to whom a tag belongs. For this purpose, a tag generates a unique pseudonym $\mathbf{p}$ and a one-time encryption key $\mathbf{k}_{\mathrm{AE}}$ for every transmission. Both are derived from the session key $\mathbf{k}_{i}$ using a key derivation function $\operatorname{KDF}(\mathbf{d}, \mathbf{k})$, where $\mathbf{d}$ denotes the key separation domain and $\mathbf{k}$ denotes the key. To be precise, $\mathbf{p}=\operatorname{KDF}\left(0, \mathbf{k}_{i}\right)$ and $\mathbf{k}_{\mathrm{AE}}=\operatorname{KDF}\left(1, \mathbf{k}_{i}\right)$. 
Messages consist of a pseudonym $\mathbf{p}$ and an encrypted payload. The latter, in turn, consists of a ciphertext $\mathbf{c}$ and a message authentication tag $\mathbf{t}$, i.e., $(\mathbf{c}, \mathbf{t})=\mathrm{AE}_{\mathbf{k}_{\mathrm{AE}}}(\mathbf{n}=\mathbf{0}, \mathbf{a}=\mathbf{p}, \mathbf{m}=\mathbf{b})$. Here, $\mathrm{AE}_{K_{\mathrm{AE}}}$ denotes authenticated encryption with associated data using key $\mathbf{k}_{\mathrm{AE}}$, where the first argument is the nonce $\mathbf{n}$, the second argument the associated data $\mathbf{a}$ (which is authenticated but not encrypted) and the third argument is the plaintext $\mathbf{m}$. Note that the nonce $\mathbf{n}$ can be set to zero as a unique key is used for every encryption. The list of beacon IDs discovered during a scan interval is denoted by $\mathbf{b}$; it always contains $q$ entries, where $q$ is a constant that is constrained by the bandwidth of the LoRa connection. If less than $q$ beacons were detected during the scan interval, the list is padded with zeroes. If more than $q$ beacons are discovered, the oldest ones are discarded.

When users download the location history data from the application server through their user terminal, the following procedure applies. First, they regenerate the current pseudonym $\mathbf{p}$ from the previous session key $\mathbf{k}_{\mathbf{i}}$ by hashing it, where $\mathbf{k}_{0}=\mathrm{H}\left(\mathbf{k}_{\text {Tag }}\right)$. Subsequently, they send a request to the application server for data $(\mathbf{c}, \mathbf{t})$ corresponding to pseudonym $\mathbf{p}$, issuing an individual request for each pseudonym. Finally, the user terminal decrypts the ciphertext c, verifies the message authentication tag $\mathbf{t}$, and processes the data items.

Since the networking service may not always be guaranteed, it is possible that messages sent by the tag are not received by the application server. In this event, the session keys $\mathbf{k}_{\mathrm{i}}$ at the user terminal and tag would be out of sync. Furthermore, when the user terminal has not been used for some period of time, its hash chain would also become desynchronized. In order to resynchronize, the user terminal advances the hash chain and queries the application server for the pseudonym derived from it, until the server again replies with a valid message. The tag's transmission interval determines an upper bound on the number of attempted requests. On initialization, the user terminal performs a similar exhaustive search to recover the session key of the first message stored on the server. However, in order to prevent timing attacks where the application server would link requests for different pseudonyms to the same user terminal, and consequently the same tag, the user terminal should issue these requests with random delays between them. As we assume the user terminal features powerful processing and high-bandwidth network connectivity, the time required for such searches is on the order of seconds, when the randomization delays are not considered.

\subsection{PUF-Based Key Generation}

For tags to remain functional after an occasional reboot, the value of the last session key $\mathbf{k}_{i}$ previously had to be stored in physically secure NVM. We now extend our design with a PUF-based key generator such that this assumption is partially mitigated. To be precise, the attacker is allowed to have read-access, and for the most part also write-access, to the NVM contents. Volatile memory, however, is still assumed to be inaccessible to the attacker. Moreover, the extended design only requires the NVM to be one-time programmable, thereby eliminating the higher manufacturing cost of multiple-time programmable NVM. Flash, for example, requires floating-gate transistors and hence additional photomasks and processing steps with respect to Complementary Metal-Oxide-Semiconductor (CMOS)-compatible fuses. 
Each tag implements an oversize PUF such that $u>1$ independent keys $\mathbf{k}_{\text {Tag }}$ can be extracted from its long response $\mathbf{x}$ [21]. For this purpose, the response $\mathbf{x}$ is subdivided into $u$ equally-sized partitions. A fuzzy extractor [31] ensures that each $\mathbf{k}_{\text {Tag }}$ can be reproduced in a reliable manner despite the inherently noisy evaluation of the PUF. This requires the storage of public helper data $\mathbf{h}$ in NVM. Each $\mathbf{k}_{\text {Tag }}$ is used for the derivation of at most $v$ session keys $\mathbf{k}_{i}$, as previously depicted in Fig. 2. After $v$ iterations, or whenever a device reboots, we move to the next partition of response $\mathbf{x}$. A monotonic counter $c \in\{1,2, \cdots, u\}$, which can be implemented with $u$ fuses, points to the currently active partition. The physical protection of this counter should primarily prohibit write-access: a rollback of $c$ would breach forward privacy. Read-access is of limited importance, given that for large-scale systems with numerous tags, $c$ is only a weak identifier. Moreover, the need for repeated physical access defeats the purpose of this attack: mostly, a user's belongings are inherently identifiable with the naked eye, and if not, it is would be easier to add a low-tech, nearly-invisible marking.

The use of $u>1$ partitions not only enables storing the session key hash chain in volatile memory, as a new chain is started when the device reboots, it also prevents side-channel attacks where a tag is repeatedly rebooted. We suggest using $u=100$ keys $\mathbf{k}_{\mathrm{Tag}} \in\{0,1\}^{128}$, given that $12.8 \mathrm{kbit}$ still fits the storage offered by a single QR code [36]. To support a device lifetime of, for example, 5 years, $v=438$ session keys $\mathbf{k}_{i}$ hence would have to derived from each $\mathbf{k}_{\text {Tag. }}$. An occasional reboot therefore shortens the lifetime with at most 18.25 days. The ESP32 microcontroller has plenty of Static Random-Access Memory (SRAM), i.e., the core component of our PUF, and NVM available, and hence induces no extra cost for the extraction of multiple keys $\mathbf{k}_{\text {Tag. }}$. On an ASIC, however, additional resources would have to allocated. Finally, we note that if the keys $\mathbf{k}_{\text {Tag }}$ are recovered by the bootloader, the boot process should be protected, e.g., by placing it in ROM.

\section{Implementation}

In Sect. 4, we proposed a system consisting of five entities. We now present its prototype on commercial hardware, thereby implementing a tag (Sect. 5.1), the application server (Sect. 5.2), and the user terminal (Sect. 5.3). Since the beacons do not require any custom functionality, we used commercial Proximity Beacons by Estimote [2]. They were configured to broadcast iBeacon packets, which contain a Universally Unique Identifier (UUID) [9]. The beacons have a configurable range of up to $70 \mathrm{~m}$. To increase their battery life, the range is set to approximately $50 \mathrm{~m}$. The use of the crowd-sourced LoRa infrastructure, managed by The Things Network [5], is not discussed in detail.

\subsection{Tag}

We selected the Pycom LoPy development board [48] to build a prototype implementation of the tag. This board features an Espressif ESP32 chipset which interfaces with Bluetooth, LoRa, and Wi-Fi radios. This chipset includes a dual-core microcontroller running at $240 \mathrm{MHz}$, and has $512 \mathrm{~KB}$ of Dynamic Random-Access Memory (DRAM) and $4 \mathrm{MB}$ of flash memory. It also has hardware accelerators for the Advanced Encryption Standard (AES) and the Secure 
Hash Algorithm (SHA) family. The board runs MicroPython, which is an implementation of the Python 3 language for microcontrollers, offering high-level APIs for a lot of its functionality (e.g., the wireless radios and crypto accelerators).

When a device boots, it connects to the LoRa network and configures two internal timers. At the firing of the first clock with period $t_{\mathrm{sci}}$, the Bluetooth radio is enabled and the tag starts scanning during a time $t_{\text {scd }}$ for iBeacon advertisement frames. When a beacon is discovered, the upper $m=4$ bytes of its UUID are stored. This enables the deployment of up to $2^{32} \approx 4.3$ billion beacons in a given area. As the beacons broadcast continuously, advertisements are received multiple times during each scan interval. Discovered identifiers are therefore only added to the transmission buffer once during each scan interval. After the scan has finished, the Bluetooth radio is turned off again. At most $q_{1}=3$ IDs are stored during each scan. In our current prototype, the scan interval $t_{\text {sci }}$ is set to five minutes, and the scan duration $t_{\mathrm{scd}}$ to one minute.

At the rate of the second, slower clock with period $t_{\mathrm{txi}}=q_{2} \cdot t_{\mathrm{sci}}$, where $q_{2}$ is a positive integer, an encrypted version $\mathbf{c}$ of the collected IDs is transmitted in addition to a pseudonym $\mathbf{p}$ and its tag $\mathbf{t}$. The latter quantities are computed from the current session key $\mathbf{k}_{\mathbf{i}}$ stored in flash memory. Afterwards, $\mathbf{k}_{\mathbf{i}+1}$ is computed and overwrites the previous session key. To prevent an attacker from linking transmissions from the same tag if they were all sent at the end of their corresponding transmission intervals, each message $(\mathbf{p}, \mathbf{c}, \mathbf{t})$ is sent at a random time within its transmission interval. An alternative solution requires all tags to be perfectly synchronized in order to transmit at the exact same global time, regardless of whether reboots occur. In our current prototype, the transmission interval $t_{\mathrm{txi}}$ to one hour, which implies $q_{2}=12$. Thus, at most $q=q_{1} \cdot q_{2}=36$ IDs need to be stored by a tag.

The size of pseudonym $\mathbf{p}$ is determined by the probability that a collision occurs between pseudonyms that belong to either the same or different users. Since the user terminal only has access to the master key $\mathbf{k}_{\text {Tag }}$ associated with its own tag, it cannot generate session keys $\mathbf{k}_{i}$ other than its own. Therefore, only messages belonging to the associated tags can be decrypted; as a consequence, collisions between pseudonyms do not leak information but increase the amount of data transmitted to and processed by the terminal. Our prototype uses eight-byte pseudonyms. Consequently, the MACs $\mathbf{t}$ should be at least 64 bits (eight bytes) to protect the messages' integrity against online attacks and to filter out such dummy traffic relating to other users. These choices result in messages of 160 bytes: $(\mathbf{p} \rightarrow 8$ bytes $)+(\mathbf{c} \rightarrow q \cdot m=144$ bytes $)+(\mathbf{t} \rightarrow 8$ bytes $)$.

Since the LoPy board features AES acceleration, we selected AES-COLM [13] as the authenticated encryption scheme. This algorithm is a candidate in the CAESAR competition and supersedes the COPA and ELmD proposals. Aside from the AES algorithm, its building blocks consist of XORs, multiplications with constants in $G F\left(2^{128}\right)$, and a linear mixing function. This makes it suited for both software and hardware implementations. Furthermore, the availability of hardware support for the SHA family of hash functions determined our choice of a hash-based key derivation function, i.e., $\operatorname{KDF}(\mathbf{d}, \mathbf{k})=\mathrm{SHA} 256(\mathbf{d} \| \mathbf{k})$. Note that our protocol depends in no way on the algorithms selected for the prototype, and that they can all be replaced with functionally identical alternatives.

The tag connects to a nearby LoRa gateway registered with The Things Network [5]. This is a global community which is building a LoRaWAN network by crowdsourcing gateways. Anyone with a compatible device can add their node 
as a gateway, while the organization provides all other backend infrastructure (e.g., servers to handle the messages received by the LoRa gateways).

SRAM PUF The extended version of our protocol requires the implementation of a PUF. As pointed out by Layman et al. [43], a Static Random-Access Memory (SRAM) can be adopted as a PUF. Its initial state $\mathbf{x} \in\{0,1\}^{n}$ after power-up provides a device-unique fingerprint. For our proof-of-concept implementation on an ESP32 microcontroller, an internal SRAM is readily available. The state $\mathbf{x}$ is read-out by listing addresses in counter mode and concatenating the corresponding 32-bit words.

Secure Sketch A secure sketch [31] provides an information-theoretically secure mechanism to transform the noisy state $X$ into a stable secret $Y$. During the enrollment, public helper data $\mathbf{h}$ is generated for a reference response $\mathbf{x}$ and is subsequently stored in flash memory. In the field, the helper data $\mathbf{h}$ allows the reference response $\mathbf{x}$, or a related variable $\mathbf{y}$, to be recovered from a newly generated response $\tilde{\mathbf{x}}$ that is sufficiently close to $\mathbf{x}$.

Instances of a secure sketch are most frequently based on a binary $[n, k, d]$ block code, where $n$ is the codeword length, $k$ is the message length, and $d$ is the minimum distance. Seven code-based constructions are known to be equivalent in terms of min-entropy loss [29]. For a uniformly distributed reference input $X$, i.e., $\mathbb{H}_{\infty}(X)=n$, it holds that $\tilde{\mathbb{H}}_{\infty}(Y \mid H)=k$. More generally, for potentially non-uniformly distributed inputs $X$, it holds that $\tilde{\mathbb{H}}_{\infty}(Y \mid H) \geq \mathbb{H}_{\infty}(X)-(n-k)$. Unfortunately, the latter bound is not very tight if the non-uniformities are major. For our prototype, we simply assume that the state $X$ is uniformly distributed, given that neither the distribution of $X$ nor the min-entropy $\mathbb{H}_{\infty}(X)$ can precisely be determined from experimental data.

Out of seven constructions, we opt for the proposal of Kang et al. [39] because of three reasons: the size of the helper data is $(n-k)$ bits rather than $n$ bits, the size of $\mathbf{y}$ is $k$ bits rather than $n$ bits, and the enrollment does not require a random number. The generator matrix is required to be in standard form, i.e., $\mathbf{G}=\left(\mathbf{I}_{k} \mathbf{P}\right)$, where $\mathbf{I}_{k}$ is the $k \times k$ identity matrix. During the enrollment, the helper data is computed as follows: $\mathbf{h} \leftarrow\left(x_{1} x_{2} \cdots x_{k}\right) \mathbf{P} \oplus\left(x_{k+1} x_{k+2} \cdots x_{n}\right)$. In the field, the reference output $\mathbf{y}=\left(x_{1} x_{2} \cdots x_{k}\right)$ is recovered as follows: $\hat{\mathbf{y}} \leftarrow \operatorname{Decode}(\tilde{\mathbf{x}} \oplus(\mathbf{0} \| \mathbf{h}))$.

We instantiate the binary code with the proposal of Van der Leest et al. [53]. To be precise, the response $\mathbf{x}$ is subdivided into $z$ partitions and the concatenation of an $\left[n_{1}=24, k_{1}=12, d_{1}=8\right]$ Golay code and an $\left[n_{2}, k_{2}=1, d_{2}=n_{2}\right]$ repetition code is applied to each partition. Globally, this can be understood as an $\left[n=z n_{1} n_{2}, k=z k_{1}, d=d_{1} d_{2}\right]$ linear code. We choose $z=11$ so that $\tilde{\mathbb{H}}_{\infty}(Y \mid H)=132$ exceeds the key size 128 . The only remaining degree of freedom, $n_{2}$, is chosen in conformity with the error rate of the PUF. The repetition decoder outputs, in addition to a presumed message $\hat{y}_{1}=x_{1}$, a level of confidence that decreases with the number of errors $\in\left\{0,1, \cdots,\left(n_{2}-1\right) / 2\right\}$ that has presumably been corrected. Subsequently, we iterate over all $2^{12}$ Golay codewords and retain the one that achieves maximum likelihood. For a bit error rate of $15 \%$ and $n_{2}=3$, i.e., a total of $n=792$ response bits, a Monte Carlo experiment shows that the failure rate for reconstructing the key $\mathbf{k}_{\mathrm{Tag}}$ is approximately $10^{-5}$. 
Robust Fuzzy Extractor To detect helper data manipulation, which might otherwise allow an attacker to recover the master key through failure statistics $[29,24]$, and to ensure that the key has been recovered correctly, we apply an integrity scheme [27]. First, we check whether the Hamming distance between the regenerated response $\tilde{\mathbf{x}}$ and the recovered response $\hat{\mathbf{x}}$ does not exceed a certain threshold. Subsequently, it is checked whether a precomputed hash value $\mathbf{h}_{\star}=\mathrm{H}_{1}(\mathbf{y}, \mathbf{h})$, which is stored in either fuses or flash, can be reproduced. Only if the latter two checks succeed, the master key is computed as $\mathbf{k}_{\text {Tag }}=\mathrm{H}_{2}(\mathbf{y}, \mathbf{h})$.

ASIC Finally, in addition to the LoPy-based design, we also propose a systemlevel architecture mapping our design to ASIC. Since tags are high-volume, low-cost, battery-powered devices, the primary design objectives are low area and low energy consumption. In the presented application scenario, a tag spends most of its lifetime in sleep mode and only occasionally performs computations or transmits messages. Therefore, the power consumption of the System-on-Chip (SoC) without communication blocks is dominated by the stand-by currents and hence not by dynamic switching behavior. Typically, in standard-cell-based designs, high area results in high static power consumption.

To avoid the high area and energy costs associated with a SHA-256 implementation, we recommend using AES-COLM for all cryptographic operations. The key $\mathbf{k}_{T a g}$ and the integrity data $\mathbf{h}_{\star}$ are computed as the tag output $\mathbf{t}$ of the authenticated encryption module, using the helper data $\mathbf{h}$ and the reference output $\mathbf{y}$ of the secure sketch as associated data $\mathbf{a}$. To be precise, $\left(-, \mathbf{k}_{T a g}\right)=\mathrm{AE}_{\mathbf{k}=\mathbf{0}}(\mathbf{n}=0, \mathbf{a}=\mathbf{y} \| \mathbf{h}, \mathbf{m}=\mathbf{0})$ and $\left(-, \mathbf{h}_{\star}\right)=\mathrm{AE}_{\mathbf{k}=\mathbf{0}}(\mathbf{n}=1, \mathbf{a}=$ $\mathbf{y} \| \mathbf{h}, \mathbf{m}=\mathbf{0})$. Ciphertexts $\mathbf{c}$ are not used and hence do not have to be computed. The values of nonce $\mathbf{n}$ can be chosen arbitrarily, but should differ for both instances. Similarly, the session key $\mathbf{k}_{i+1}$, the pseudonym $\mathbf{p}$, and the encryption key $\mathbf{k}_{\mathrm{AE}}$ are derived using three different values of the nonce $\mathbf{n}$. To be precise, $\left(-, \mathbf{k}_{i+1}\right)=\mathrm{AE}_{\mathbf{k}_{i}}(\mathbf{n}=1, \mathbf{a}=\mathbf{0}, \mathbf{m}=\mathbf{0}),(-, \mathbf{p})=\mathrm{AE}_{\mathbf{k}_{i}}(\mathbf{n}=2, \mathbf{a}=\mathbf{0}, \mathbf{m}=\mathbf{0})$, and $\left(-, \mathbf{k}_{\mathrm{AE}}\right)=\mathrm{AE}_{\mathbf{k}_{i}}(\mathbf{n}=3, \mathbf{a}=\mathbf{0}, \mathbf{m}=\mathbf{0})$.

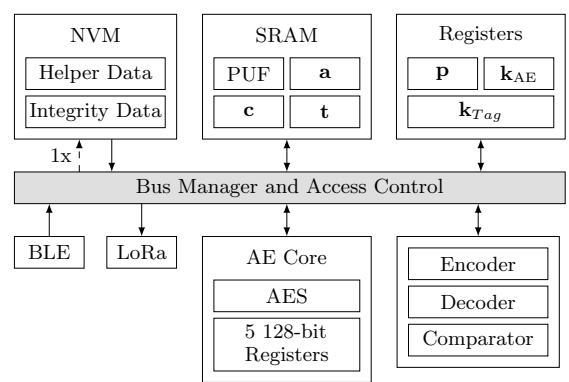

Fig. 3: System-level design of a prospective ASIC.

Fig. 3 shows the architecture of a tag. The system contains an embedded SRAM to store the beacon identifiers and the encrypted message before the transmission. Its power-up state serves as the PUF response $\mathbf{x}$. Each PUF response is generated using 132 bytes of raw SRAM data. A 128 kb SRAM 
block is sufficient for generating 100 PUF responses. In addition, the tag uses a one-time programmable NVM to store the helper data and the integrity data. The required capacity of this memory is $10.6 \mathrm{kB}$. A AES-COLM core consists of the round-based AES-128 module, data paths for linear operations and five 128-bit registers for storing intermediate results. Flip-flop-based registers are used for storing the session key, the pseudonym and the encryption key. Dedicated data paths are used for encoding and decoding the PUF responses and for the integrity check. The bus manager controls bus access and communication between the cores and networking blocks. A Finite State Machine (FSM) is used to execute the protocol. In order to reduce the leakage power, the system should also contain power management logic for switching off the cores during sleep mode.

\subsection{Application Server}

After receiving a message from the tag through one of its gateways, the LoRa infrastructure provider forwards the payload to the application server over HTTP. The application server therefore runs a simple web service that accepts the messages and stores them in a database. It also provides an endpoint where the user terminal can retrieve the message corresponding to a specific pseudonym $\mathbf{p}$. In addition, the application server stores the geographical coordinates of each beacon, which can be requested from a second endpoint.

The application server was built as a REST-based web service, which can be queried through a JSON API. The service was implemented in Go, with all data being stored in Bolt [1], a high-performance key/value store. Since both the messages and beacon coordinates are always accessed through their keys, i.e., the pseudonym and beacon identifier respectively, this database was preferred over more traditional relational databases. In order to reduce storage cost, the server periodically removes messages from its database (e.g., every month).

\subsection{User Terminal}

We implemented a prototype of the terminal as an iOS app written in Swift. Both AES-COLM and our KDF based on SHA-256 could be easily implemented for this platform. The performance of these operations is of lesser concern here, as our messages are relatively short and smartphones have significantly more processing power than tags. When the app is started for the first time, access is requested to the phone's camera to scan a QR code containing the tag key $\mathbf{k}_{\text {Tag }}$, after which it is stored in iOS' keychain. Recall that this process links the terminal to the tag, allowing it to reconstruct the sequence of pseudonyms which can be used to retrieve messages from the server. The app will then synchronize its session key $\mathbf{k}_{\mathrm{i}}$ by repeatedly querying the server until no new message is found. In order to preserve forward privacy, the terminal will always update the iOS keychain with the last synchronized session key, as this prevents an attacker from reconstructing the hash chain if they manage to breach the app. As mentioned in Sect. 3, protecting forward privacy if the terminal is compromised, requires that the user sets limits on the history kept by the user terminal. Configuring the user terminal to remove data immediately after displaying it would fully guarantee forward privacy, at the cost of not having access to location history.

When launched, the app retrieves all messages from the application server since its previous run: it repeatedly advances the session key hash chain and 
calculates the corresponding pseudonym $\mathbf{p}$ until the server responds that no data is available. The app will also retrieve the public list of beacons and their geographical location from the server. After decrypting each message, the beacon identifiers it contains are matched to the coordinate list and the tag's position during the scan interval is calculated through interpolation. Its location during the last 24 hours is then shown on a map. In addition, the user can view the tag's calculated location during each scan interval.

\section{Analysis}

We first present a security and privacy analysis of our design, referencing the requirements identified in Sect. 4.2. Next, we discuss how our design intrinsically protects against side-channel attacks (Sect. 6.2).

\subsection{Security and Privacy}

Confidentiality and Authenticity of Location Data (Satisfies S1, S2, and S3). A tag's location data is always sent in encrypted form using a secret key $\mathbf{k}_{\mathrm{AE}}$ that is known only to the tag and an authorized user terminal. This offers end-to-end confidentiality, as it prevents intermediate entities from learning anything about the tag's location. Due to the use of an authenticated encryption scheme, unauthorized modifications are detected with extremely high probability.

Tag Identity Privacy (Satisfies P1). To hide its identity, a tag appends a pseudonym $\mathbf{p}$ and hence not a public identifier to each message. This pseudonym $\mathbf{p}$ is generated using a KDF and a session key $\mathbf{k}_{i}$ that is known only to the tag and the authorized user terminal. Since $\mathbf{p}$ is relatively short, an occasional collision could imply that user A inadvertently downloads the encrypted location data of user B. Note, however, that user A cannot successfully decrypt user B's data.

Message Unlinkability (Satisfies P2): Due to the freshness of the pseudonyms $\mathbf{p}$, an unauthorized entity cannot link two or more messages that have been sent by the same tag. Furthermore, all LoRa messages have the same size and are sent at random intervals to prevent meta-data and traffic analysis attacks. An anonymous channel, e.g., Tor, between each user terminal and the application server prevents the latter from linking pseudonyms $\mathbf{p}$ of which a single terminal requests the location data.

Forward privacy (Satisfies P3): Since only the last session key $\mathbf{k}_{i}$ is stored in the secure NVM, if adversaries compromise a tag or a user terminal, they cannot compute old session keys to learn the tag's past locations.

\subsection{Side-Channel Security}

An adversary who obtains physical access to a tag could mount a side-channel attack in an attempt to recover its cryptographic keys. This involves measuring the tag's instantaneous power consumption (or any related magnitude such as electromagnetic field intensity) while it performs a cryptographic operation. We focus on two common side-channel attacks: SPA and DPA. The protocol described in Sect. 4.3 features an intrinsic countermeasure. By construction, the protocol limits the number of cryptographic executions that an adversary may ask the 
device to perform under the same key. This limits the information leaked by each key, so that if the key is changed often enough, it becomes unfeasible for an adversary to reconstruct the whole key. This is the main working principle of "fresh re-keying" systems, initiated independently in the seminal patent of Kocher [41] and $[26, \S 6.6]$, and continued in $[45,44]$.

More concretely, fresh re-keying guarantees resistance to SPA and DPA based on the following two assumptions [45]. Firstly, the key update mechanism must be resistant to DPA and SPA. In our case, the key update mechanism is $\mathbf{k}_{i+1}=\mathrm{H}\left(\mathbf{k}_{i}\right)$. This makes DPA impossible, since each value of the hash chain is handled only once. Given that session key $\mathbf{k}_{i}$ is stored in NVM, a reboot does not allow an attacker to remeasure traces. The resistance to SPA must be guaranteed by constant-time, constant-flow code and a noisy enough hardware platform.

Secondly, the pseudonym and encryption key generation $\mathrm{G}\left(\mathbf{k}_{i}\right)$ must be resistant to SPA. This is again guaranteed by constant-time, constant-flow code and a noisy enough hardware platform. Note that this makes the protocol "somewhat stateful", since the receiving end must keep a minimal state as the last seen key from the hash chain. Nevertheless, we believe that this is an attractive property of the protocol since it protects forward privacy: key breaches do not allow to retroactively de-anonymize previous messages (and thus learn the past locations of the device). Thus, we fulfill design requirement P3 from Sect. 4.2.

For the extended version of our protocol, it is expected that the fuzzy extractor is resistant to first-order DPA attacks as well. Not only is the SRAM partition regularly updated, the monotonic counter prevents traces from being remeasured after a reboot. The masking-based countermeasure of Merli et al. [46], which precludes DPA attacks on a secure sketch, is therefore omitted. More sophisticated attacks such as template attacks [40] are ruled out since they require a characterization of the device leaking behaviour.

\section{Evaluation}

We evaluate our prototype on Pycom's LoPy board (Sect. 5) in terms of latency in Sect. 7.1. Subsequently, we quantify its power/energy usage in Sect. 7.2 through a custom-designed measurement board for Universal Serial Bus (USB)-powered devices. In Sect. 7.3, we quantify the randomness and stability of its SRAM PUF. Finally, we estimate the energy usage of our ASIC design in Sect. 7.4.

\section{$7.1 \quad$ Latency}

The energy usage, which is the dominant constraint in our application, scales to some extent proportionally with the latency of the cryptographic operations on our microcontroller. First, we benchmarked the derivation of the pseudonym $\mathbf{p}$ and the encryption key $\mathbf{k}_{\mathrm{AE}}$ : this operation takes on average $855.21 \mu \mathrm{s}$. Next, our COLM implementation averages $96.13 \mathrm{~ms}$ when encrypting a single 144-byte list $\mathbf{b}$ of beacon IDs (Sect. 5.1).

\subsection{Power and Energy}

In addition to the tag's performance, we evaluated its power and energy usage. To this end, we designed a USB pass-through board, powering the LoPy through 
this board, rather than directly from the power source. A $1 \Omega$ resistor was placed between the source's ground line and the Lopy's ground, allowing us to measure the current drawn by the board by sampling the voltage over this resistor. The measurement points were connected to an MSP430F5529 development board, which features a 12-bit Analog-to-Digital Converter (ADC) (cf. Fig. 4). As the LoPy is rated to draw at most $400 \mathrm{~mA}$, the microcontroller's REF module was used to generate a $1.5 \mathrm{~V}$ reference voltage, yielding a measurement resolution of $3.66 \mathrm{~mA}$, as the measured voltage is equal to current due to the use of a $1 \Omega$ resistor. Since a USB port is used as the source, the board is powered at $5 \mathrm{~V}$.

Fig. 4: Measurement setup for the power usage of our prototype implementation. In addition to the USB pass-through board connected to an MSP430F5529 (bottom left) and LoPy (bottom right), two Estimote beacons are shown.

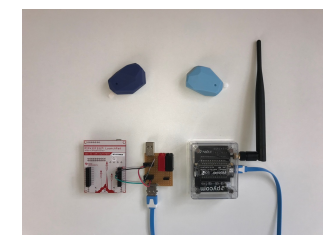

Before measuring the energy usage of our overall application, we looked at the board's power usage in four different instances. First, we measured that the device draws $39.25 \mathrm{~mA}$ when idling with all radios asleep. Placing the board in deep sleep mode reduced the average current to $19.87 \mathrm{~mA}$, which is still much higher than expected due to a silicon bug in our version of the LoPy. ${ }^{1}$ Next, we programmed the board to perform one million AES encryptions of a single block in ECB mode and to hash 16 bytes one million times, measuring respective average energy usages of $35.58 \mu \mathrm{J}$ per encryption and $44.17 \mu \mathrm{J}$ per hash.

Lastly, we investigated the current usage when our protocol implementation is running on the board. During a one-hour interval with a single LoRa transmission, $874.29 \mathrm{~J}$ is consumed on average. As can be seen from Fig. 5, this is mainly caused by the Bluetooth scanning, with the board drawing $88.16 \mathrm{~mA}$ during the scan interval, because the current ESP32 SDK only supports enabling both the BLE and Classic modes of the Bluetooth radio. Note that our implementation does not place the board in deep sleep, resulting in high average power usage. However, it can be seen from the graph that the device is only active for short bursts, and the required energy to run our protocol would therefore drop significantly when less current is drawn in between. Extending the prototype implementation to make use of deep sleep functionality is left as future work. Due to the high Bluetooth current usage caused by the ESP32 SDK, our prototype implementation runs for about 103 hours when used with a $5000 \mathrm{~mA}$ h battery. However, note that the battery life of our application can be improved by tweaking the scan duration and scan interval. For example, the energy usage over the one-hour interval drops to $718.55 \mathrm{~J}$ when $t_{s c i}$ and $t_{s c d}$ are configured to 15 minutes and 15 seconds respectively. Finally, the development of an ASIC, rather than a general-purpose chip, greatly reduces current consumption, enabling the use of coin-sized batteries (Sect. 7.4).

The listed issues with the current prototype complicate direct energy comparison with GPS and RFID (Sect. 2). Furthermore, the evaluated prototype is a

\footnotetext{
${ }^{1}$ https://forum.pycom.io/topic/1022/root-causes-of-high-deep-sleep-current
} 
SoC featuring several components in addition to the BLE receiver. Additionally, as shown by our analysis, the energy usage very much depends on the exact implementation and the activity of each component. However, while we did not evaluate the tracking accuracy and range of our design, it performs better than RFID in these areas through its use of BLE, but is outperformed by GPS.

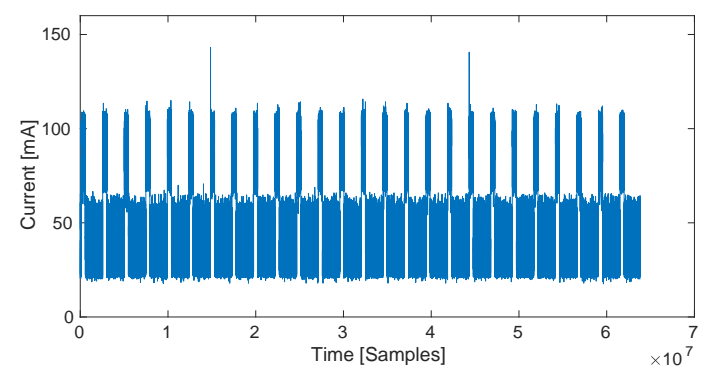

Fig. 5: The LoPy's current usage $(20-140 \mathrm{~mA})$ when running the prototype of the protocol implementation during two hours of operation, with the graph starting halfway through the scan interval.

\subsection{SRAM PUF}

Additionally, we have evaluated whether the LoPy's SRAM could be used for PUFbased key generation. The nominal environment, during which the enrollment takes place, is defined as $T=25^{\circ} \mathrm{C}$. The error rate between two read-outs, and averaged over $n=10^{6}$ response bits, is $6.1 \%$. In the field, however, different temperatures might apply, thereby deteriorating the reproducibility of the state $\mathbf{x}$. Using an ESPEC SH-662 temperature chamber, we evaluated the PUF of a single device under an extremely cold, i.e., $T=-40^{\circ} \mathrm{C}$, and an extremely hot, i.e., $T=85^{\circ} \mathrm{C}$, environment. The error rates with respect to the nominal environment are $16.8 \%$ and $11.2 \%$ respectively. Although the latter two error rates are higher than for the $65 \mathrm{~nm}$ CMOS ASIC of Koeberl et al. [42], we emphasize that the ESP32 is only a prototype device. A temporal majority vote, which could have lowered the error rates at the cost of multiple read-outs, was not implemented.

Given that an SRAM consists of ideally autonomous cells, the distribution of $X$ has the potential to be fairly uniform over $\{0,1\}^{n}$. For a set of 10 devices and $n=10^{6}$, we apply statistical tests that can detect non-uniformities. To be precise, we estimate several probabilities through a $95 \%$ confidence interval and check whether their ideal value $50 \%$ is enclosed. The interval for the probability that a given response bit of a given device equals 1 is [50.01\%,50.07\%]. The interval for the probability that two neighboring response bits in a given word of a given device are equal is $[50.44 \%, 50.50 \%]$. The interval for the probability that a given bit in two consecutive words of a given device is equal is [49.71\%, 49.77\%]. The interval for the probability that two devices produce the same value for a given response bit is $[50.24 \%, 50.27 \%]$. We conclude that minor non-uniformities are likely to exist. 


\subsection{ASIC}

In order to estimate the battery lifespan for the future ASIC implementation we look into the energy consumption of the cryptographic core and communication blocks. The energy cost of a round-based AES-128 implementation is $350.7 \mathrm{pJ}$ per encryption using the STM $90 \mathrm{~nm}$ CMOS technology [23]. Every hour, 35 AES encryptions are required for preparing the message and updating the key, which costs $12.3 \mathrm{~nJ}$. According to a study presented in [51], the energy cost of a $128 \mathrm{~kb}$ SRAM using 32-bit words and manufactured in $90 \mathrm{~nm}$ CMOS technology is less than $10 \mathrm{pJ}$ per access.

For SRAM using 32-bit word lengths, the presented protocol requires approximately 760 memory accesses per hour, which consumes less than $7.6 \mathrm{~nJ}$. The transmission of each LoRa message takes $2 \mu \mathrm{s}$. Based on the experiments using the LoPy, the driving current during message transmission is $55.2 \mathrm{~mA}$. This results in a LoRa communication cost of $55.2 \mu \mathrm{J}$ per hour. For BLE communication, we estimate the energy consumption based on a Texas Instruments Bluetooth controller [10]. From the number of scanning intervals we approximate that BLE consumes $2.8 \mathrm{~mJ}$ every hour. The energy consumption of the chip will be dominated by the BLE communication energy and static leakage. In order to estimate battery lifetime, we use the Energizer CR1620 coin-sized battery [3]. Approximating the energy consumption by the BLE leakage power in shutdown mode and the transmission power, we end up with a rough battery lifetime approximation of 74 months.

\section{Conclusion}

This paper presented the design and a prototype implementation of a privacyfriendly tracking system that builds upon the following IoT technologies: inexpensive tags, Bluetooth Low Energy (BLE), and LoRa communications. Only legitimate tag owners can track their devices, while no information is leaked to network operators or service providers. The system was evaluated through a prototype based on an off-the-shelf IoT node. We have also produced estimates for the energy and power consumption of a possible ASIC taking the role of the tag. We have demonstrated that secure key storage using an SRAM PUF and side-channel resistance is achievable within realistic area and energy budgets. Future work should focus on a detailed ASIC design and a prototype for a privacy-friendly variant of the LoRa protocol with changing MAC addresses. The automated detection of compromised beacons could be added to the demonstrator. A large-scale field test would be helpful to obtain a more thorough experimental validation of the resistance against traffic analysis.

\section{Acknowledgements}

We would like to thank the anonymous reviewers for their feedback, as well as Patrick Tague for acting as our shepherd. This work is the result of collaborative research partially funded by the Attached Institute of ETRI. It was also supported in part by the KU Leuven Research Council through C16/15/058, the European Union's Horizon 2020 research and innovation programme under grant agreements No 644052 HECTOR and No 644371 WITDOM, ERC Advanced Grant 695305. Pieter Maene is an SB PhD fellow at Research Foundation - Flanders (FWO). 


\section{References}

1. Bolt. https://github.com/boltdb/bolt.

2. Estimote. https://estimote.com.

3. Product Datasheet Energizer CR1620. http://data.energizer.com/pdfs/cr1620. pdf. Accessed: 2017-07-01.

4. RAPID7. https://community.rapid7.com/community/infosec/blog/2016/10/ 25/multiple-bluetooth-low-energy-ble-tracker-vulnerabilities.

5. The Things Network. https://thethingsnetwork.org.

6. Tile. https://www. thetileapp.com.

7. TrackR. https://thetrackr.com/bravo.

8. A2235-H Stack-up Antenna SiRFstarIV Integrated Solution. Datasheet, Maestro, 2012.

9. Proximity Beacon Specification. Specification, Apple, 2015.

10. CC256x Dual-Mode Bluetooth Controller (Rev. E). Datasheet, 2016.

11. SL3S1214 UCODE 7m Rev. 3.3. Datasheet, NXP Semiconductors, 2016.

12. B. Alomair, A. Clark, J. Cuellar, and R. Poovendran. Scalable rfid systems: A privacy-preserving protocol with constant-time identification. IEEE Transactions on Parallel and Distributed Systems, 23(8):1536-1550, Aug 2012.

13. E. Andreeva, A. Bogdanov, N. Datta, A. Luykx, B. Mennink, M. Nandi, E. Tischhauser, and K. Yasuda. COLM v1. https://competitions.cr.yp.to/round3/ colmv1.pdf, 2016.

14. G. Avoine. Privacy Issues in RFID Banknote Protection Schemes. In 6th International Conference on Smart Card Research and Advanced Applications, pages 33-48, 2004.

15. G. Avoine. Privacy Challenges in RFID. In J. García-Alfaro, G. Navarro-Arribas, N. Cuppens-Boulahia, and S. D. C. di Vimercati, editors, 6th and 4th International Workshop Data Privacy Management and Autonomous Spontaneous Security, volume 7122 of Lecture Notes in Computer Science, pages 1-8. Springer, 2011.

16. G. Avoine, A. Beaujeant, J. Hernandez-Castro, L. Demay, and P. Teuwen. A Survey of Security and Privacy Issues in ePassport Protocols. ACM Comput. Surv., 48(3):47:1-47:37, 2016.

17. G. Avoine, M. A. Bingöl, X. Carpent, and S. B. O. Yalcin. Privacy-Friendly Authentication in RFID Systems: On Sublinear Protocols Based on Symmetric-Key Cryptography. IEEE Trans. Mob. Comput., 12(10):2037-2049, 2013.

18. G. Avoine, I. Coisel, and T. Martin. Untraceability Model for RFID. IEEE Trans. Mob. Comput., 13(10):2397-2405, 2014.

19. G. Avoine and P. Oechslin. A Scalable and Provably Secure Hash-Based RFID Protocol. In 3rd IEEE Conference on Pervasive Computing and Communications Workshops, pages 110-114, 2005.

20. G. Avoine and P. Oechslin. RFID Traceability: A Multilayer Problem. In 9th International Conference Financial Cryptography and Data Security, pages 125-140, 2005.

21. A. Aysu, E. Gulcan, D. Moriyama, P. Schaumont, and M. Yung. End-to-end design of a PUF-based privacy preserving authentication protocol. In T. Güneysu and H. Handschuh, editors, 17th Workshop on Cryptographic Hardware and Embedded Systems (CHES 2015), volume 9293 of Lecture Notes in Computer Science, pages 556-576. Springer, Sept. 2015.

22. L. L. B. Alomair and R. Poovendran. Securing low-cost rfid systems: an unconditionally secure approach, 2010.

23. S. Banik, A. Bogdanov, T. Isobe, K. Shibutani, H. Hiwatari, T. Akishita, and F. Regazzoni. Midori: A Block Cipher for Low Energy. In 21st International Conference on Advances in Cryptology, pages 411-436, 2015. 
24. G. T. Becker. Robust Fuzzy Extractors and Helper Data Manipulation Attacks Revisited: Theory vs Practice. Cryptology ePrint Archive, Report 2017/493, 2017. http://eprint.iacr.org/2017/493.

25. A. Bochem, K. Freeman, M. Schwarzmaier, O. Alfandi, and D. Hogrefe. A Privacypreserving and Power-efficient Bicycle Tracking Scheme for Theft Mitigation. In 2nd IEEE International Conference on Smart Cities, pages 1-4, 2016.

26. J. Borst. Block Ciphers: Design, Analysis and Side-Channel Analysis. PhD thesis, Katholieke Universiteit Leuven, 2001. Bart Preneel and Joos Vandewalle (promotors).

27. X. Boyen, Y. Dodis, J. Katz, R. Ostrovsky, and A. Smith. Secure remote authentication using biometric data. In R. Cramer, editor, Advances in Cryptology EUROCRYPT 2005, volume 3494 of Lecture Notes in Computer Science, pages 147-163. Springer, May 2005.

28. B. Danev, D. Zanetti, and S. Capkun. On Physical-layer Identification of Wireless Devices. ACM Comput. Surv., 45(1):6:1-6:29, 2012.

29. J. Delvaux. Security Analysis of PUF-Based Key Generation and Entity Authentication. PhD thesis, KU Leuven, June 2017.

30. R. Dingledine, N. Mathewson, and P. F. Syverson. Tor: The Second-Generation Onion Router. In 13th USENIX Security Symposium, pages 303-320, 2004.

31. Y. Dodis, R. Ostrovsky, L. Reyzin, and A. Smith. Fuzzy extractors: How to generate strong keys from biometrics and other noisy data. SIAM Journal on Computing, 38(1):97-139, Mar. 2008.

32. A. Hassidim, Y. Matias, M. Yung, and A. Ziv. Ephemeral Identifiers: Mitigating Tracking \& Spoofing Threats BLE Beacons. 2016.

33. D. Henrici, J. Götze, and P. Müller. A Hash-based Pseudonymization Infrastructure for RFID Systems. In 2nd International Workshop on Security, Privacy and Trust in Pervasive and Ubiquitous Computing, pages 22-27, 2006.

34. D. Henrici and P. Müller. Hash-based Enhancement of Location Privacy for RadioFrequency Identification Devices using Varying Identifiers. In 2nd IEEE Conference on Pervasive Computing and Communications Workshops, pages 149-153, 2004.

35. D. Henrici and P. Müller. Providing Security and Privacy in RFID Systems Using Triggered Hash Chains. In 6th Annual IEEE International Conference on Pervasive Computing and Communications, pages 50-59, 2008.

36. Information - Automatic identification and data capture techniques - QR Code barcode symbology specification. Standard, International Organization for Standardization, 22015.

37. A. Juels and R. Pappu. Squealing Euros: Privacy Protection in RFID-Enabled Banknotes. In 7 th International Conference on Financial Cryptography, pages 103-121, 2003.

38. A. Juels, R. L. Rivest, and M. Szydlo. The Blocker Tag: Selective Blocking of RFID Tags for Consumer Privacy. In 10th ACM Conference on Computer and Communications Security, pages 103-111, 2003.

39. H. Kang, Y. Hori, T. Katashita, M. Hagiwara, and K. Iwamura. Cryptographic key generation from PUF data using efficient fuzzy extractors. In 16th International Conference on Advanced Communication Technology, pages 23-26. IEEE, Feb. 2014.

40. D. Karakoyunlu and B. Sunar. Differential template attacks on PUF enabled cryptographic devices. In 2nd Workshop on Information Forensics and Security (WIFS 2010), pages 1-6. IEEE, Dec. 2010.

41. P. Kocher. Leak-resistant Cryptographic Indexed Key Update, 2003. US Patent $6,539,092$.

42. P. Koeberl, R. Maes, V. Rožić, V. van der Leest, E. Van der Sluis, and I. Verbauwhede. Experimental Evaluation of Physically Unclonable Functions in $65 \mathrm{~nm}$ CMOS. In 38th European Conference on Solid-State Circuits, pages 486-489, Sept. 2012 . 
43. P. A. Layman, S. Chaudhry, J. G. Norman, and J. R. Thomson. Electronic fingerprinting of semiconductor integrated circuits, May 2004. US Patent 6738294.

44. M. Medwed, C. Petit, F. Regazzoni, M. Renauld, and F. Standaert. Fresh Re-keying II: Securing Multiple Parties against Side-Channel and Fault Attacks. In 10th International Conference on Smart Card Research and Advanced Applications, pages 115-132, 2011.

45. M. Medwed, F. Standaert, J. Großschädl, and F. Regazzoni. Fresh Re-keying: Security against Side-Channel and Fault Attacks for Low-Cost Devices. In 3rd International Conference on Cryptology in Africa, pages 279-296, 2010.

46. D. Merli, F. Stumpf, and G. Sigl. Protecting PUF Error Correction by Codeword Masking. Cryptology ePrint Archive, Report 2013/334, 2013. http://eprint.iacr. org/2013/334.

47. D. Molnar and D. A. Wagner. Privacy and Security in Library RFID: Issues, Practices, and Architectures. In 11th ACM Conference on Computer and Communications Security, pages 210-219, 2004.

48. Pycom. LoPy. https://www.pycom.io/product/lopy/.

49. T. Ristenpart, G. Maganis, A. Krishnamurthy, and T. Kohno. Privacy-Preserving Location Tracking of Lost or Stolen Devices: Cryptographic Techniques and Replacing Trusted Third Parties with DHTs. In 17th USENIX Security Symposium, pages 275-290, 2008.

50. J. Saito, J. Ryou, and K. Sakurai. Enhancing Privacy of Universal Re-encryption Scheme for RFID Tags. In 2nd International Conference on Embedded and Ubiquitous Computing, pages 879-890, 2004.

51. V. Sharma, S. Cosemans, M. Ashouie, J. Huisken, F. Catthoor, and W. Dehaene. Ultra Low-Energy SRAM Design for Smart Ubiquitous Sensors. IEEE Micro, 32(5):10-24, 2012.

52. S. Spiekermann and O. Berthold. Maintaining Privacy in RFID-enabled Environments. Privacy, Security and Trust within the Context of Pervasive Computing, pages 137-146, 2005.

53. V. van der Leest, B. Preneel, and E. van der Sluis. Soft decision error correction for compact memory-based PUFs using a single enrollment. In E. Prouff and P. Schaumont, editors, 14th Workshop on Cryptographic Hardware and Embedded Systems (CHES 2012), volume 7428 of Lecture Notes in Computer Science, pages 268-282. Springer, Sept. 2012.

54. S. A. Weis, S. E. Sarma, R. L. Rivest, and D. W. Engels. Security and Privacy Aspects of Low-Cost Radio Frequency Identification Systems. In 1st International Conference on Security in Pervasive Computing, pages 201-212, 2003. 